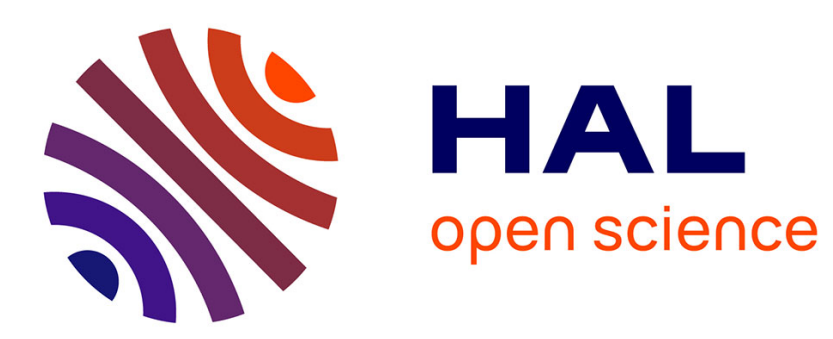

\title{
Influence of concrete fracture on the rain infiltration and thermal performance of building facades
}

\author{
Simon Rouchier, Monika Woloszyn, Geneviève Foray, Jean-Jacques Roux
}

\section{To cite this version:}

Simon Rouchier, Monika Woloszyn, Geneviève Foray, Jean-Jacques Roux. Influence of concrete fracture on the rain infiltration and thermal performance of building facades. International Journal of Heat and Mass Transfer, 2013, 61, pp.340-352. 10.1016/j.ijheatmasstransfer.2013.02.013 . hal-00798374v2

\section{HAL Id: hal-00798374 \\ https://hal.science/hal-00798374v2}

Submitted on 9 Apr 2013

HAL is a multi-disciplinary open access archive for the deposit and dissemination of scientific research documents, whether they are published or not. The documents may come from teaching and research institutions in France or abroad, or from public or private research centers.
L'archive ouverte pluridisciplinaire HAL, est destinée au dépôt et à la diffusion de documents scientifiques de niveau recherche, publiés ou non, émanant des établissements d'enseignement et de recherche français ou étrangers, des laboratoires publics ou privés. 


\title{
Influence of concrete fracture on the rain infiltration and thermal performance of building facades
}

\author{
Simon Rouchier*1 ${ }^{*}$ Monika Woloszyn ${ }^{2}$, Geneviève Foray ${ }^{3}$, and Jean-Jacques Roux ${ }^{4}$ \\ ${ }^{1}$ CETHIL CNRS UMR5008, MATEIS CNRS UMR5510, INSA-Lyon, Université Lyon 1, F-69621 Villeurbanne, France \\ ${ }^{2}$ LOCIE, CNRS-UMR5271, Université de Savoie, Campus Scientifique, Savoie Technolac, 73376 Le Bourget-du-Lac Cedex, France \\ ${ }^{3}$ INSA-Lyon, MATEIS CNRS UMR5510, F-69621 Villeurbanne, France \\ ${ }^{4}$ CETHIL, CNRS UMR5008, INSA-Lyon, Université Lyon 1, Bât. S. Carnot, 9 Rue de la Physique, F-69621 Villeurbanne, France
}

Postprint: Rouchier S., Woloszyn M., Foray G., Roux J.-J., 2013. Influence of concrete fracture on the rain infiltration and thermal performance of building facades, International Journal of Heat and Mass Transfer vol. 61, pp. 340-352. DOI: 10.1016/j.ijheatmasstransfer.2013.02.013

\begin{abstract}
Water infiltration is known to play an important part in the degradation process of construction materials. Over time, microscopic and macroscopic cracks progressively develop under the effects of mechanical loading and sorption/desorption cycles: their influence is to be accounted for in long-term hygrothermal performance assessments of the building envelope. The present work aims at showing the potential consequences of cracking on the heat and moisture transfer across building facades, in order to justify the need for the identification of damage to prevent durability and thermal issues. Specific simulation cases of insulated and non-insulated building facades were defined, and submitted to atmospheric boundary conditions for simulation times of one month. Some of the simulation geometries included previous measurements of crack patterns in concrete. The comparison of fractured and non-fractured building facades showed the effects of cracks on the moisture accumulation and thermal performance of these wall configurations, thus giving an estimate of what these effects might be in real conditions. A methodology is thus proposed for the identification of renovation needs, which may be applied for the purpose of durability assessments as well.
\end{abstract}

Keywords heat; moisture; modelling; building material; fracture

\section{Introduction}

Many causes for the degradation of building materials are carried by moisture. Indeed, infiltrated water may transport chemicals, alter mechanical properties and cause freeze thaw damage or mould development. It may also affect thermal properties and overall efficiency, as well as the health and comfort of the occupants. Over time, the pore structure of such materials may however be altered by cracks and defects caused by mechanical loading and aggravated by moisture-induced degradation. All sizes of fractures may have a strong impact on heat and moisture flow in the building envelope, and their influence is to be accounted for in any long-term performance assessment.

Hygrothermal simulations of building components are now commonly applied to oriented design [20] and to the evaluation of moisture related concerns. Examples include the assessment of the risk of mould growth [34], of moisture buffering in construction materials [1], or of moisture related damage [39]. Hygrothermal modelling

*Electronic address: simon.rouchier@insa-lyon.fr ; Corresponding author 
has also been the target of numerical optimisation [13], and several codes are now commercialy available [21, 9]. HAM (Heat, Air and Moisture) simulation codes however often rely on the assumption that material properties are constant over time and do not explicitly allow including the effects of material ageing.

A considerable amount of work has already been performed for the prediction of moisture flow in fractured porous media. Two main approaches can be distinguished. The first method is a fully coupled scheme, in which the same finite-element mesh is used for flow and transport modelling in both the fracture and the porous network. The FE mesh must be adapted to the geometry of the cracks [22], and strategies have been proposed for its automated refinement with crack propagation [32]. Segura and Carol [35] assigned a type of double-nodded, zero-thickness elements to the crack, allowing an explicit formulation of longitudinal and transverse flows, and of exchange terms between the porous medium and the fracture. They later used this formulation for the expression of a fully coupled hydro-mechanical model [36] for the prediction of flow in fracturing geomaterials. The second method is a staggered approach, in which the transport equations for flow and transport are iteratively solved in the porous medium and in the fracture. Between each iteration, the capillary pressure corresponding to the calculated pressure field in the crack is imposed as boundary condition at the matrix-fracture interface. This approach was followed by Roels et al. [26, 25], who followed the progress of the moisture front in a fracture by combining a quasi-static pressure equation and a Darcian flux equation. An important asset of the staggered approach is the fact that it allows emancipating the FE mesh from the crack geometry: the extended (or generalised) finite element method (XFEM), based on the partition of unity method [16], allows including discontinuities in the problem field by adding degrees of freedom to a set of nodes surrounding the cracks [8, 42]. This method allows accounting for crack propagation without modification of the mesh [17]. It was applied in recent studies for the modelling of hygrothermal damage processes [18], moisture uptake [2] and heat transfer [19] in fractured porous media. Both monolithic and staggered approaches allow accounting for moving fractures and including all coupled hydromechanical effects at the interfaces between fractures and matrix. Some authors argue that the staggered approach is more effective in terms of computational costs [26, 18, 2], although Segura and Carol found the monolithic approach more appropriate in case of strong coupling or non-linear discontinuity behaviour [37].

In the current state of research, long-term simulations of building components do not include the effects of material ageing. Inversely, current applications of full hygro-thermo-mechanical modelling often lie on the manual input of fractures in the problem field [22, 26, 2, 19, 37, 23], which does not reflect the complexity of real crack patterns. Indeed, a numerical simulation of the ageing of a building component over years of service life, including coupled hydromechanical effects and progressive damage modelling, would require an extensive knowledge of all environmental factors which may influence material degradation. The above mentioned cases are suitable for the service life prediction of construction materials. A complementary approach is however preferable for the hygrothermal performance estimation of existing, already damaged building components.

The present paper is part of a recently undertaken project, aimed at integrating the effects of damage in heat and moisture transfer simulations at the scale of building components. A mixed approach is followed to this aim, using experimental measurements of crack patterns as an input for a numerical model for coupled heat and moisture flow. In a recent study [28], digital image correlation and acoustic emission monitoring were found reliable for the quantification and localisation of all scales of fissures, from micro-cracks to macroscopic fractures. A numerical code was then developed [31], that may include these measurements into a finite-element frame for the prediction of coupled heat and moisture flow. The next step of this procedure, and topic of the present paper, is the application of this simulation code in order to estimate the consequences of cracking on the moisture accumulation and thermal performance of building facades, subjected to realistic climatic conditions during long periods of time. Specific simulation cases of insulated and non-insulated building facades were defined, and submitted to atmospheric boundary conditions for simulation times of one month. Some of the simulation geometries included previous measurements of crack patterns in concrete. The comparison of fractured and non-fractured building facades aim at showing the potential effects of cracks on the moisture accumulation and thermal performance of these wall configurations, thus giving an estimate of what these effects might be in real conditions.

The model for heat and moisture transfer in fractured porous media is first described in Sec. 2: the balance equations are written on the basis on simplifying hypotheses, and the conditions of their implementation into a finite-element frame are explained. This initial model was validated on the basis of the Hamstad benchmark package. We then describe how fractures are integrated into the geometry of the problem and how their influence 
on the flow is accounted for. Then, Sec. 3 clarifies the questioning of the study and shows which simulation cases were defined in order to answer it. These simulation settings are entirely defined in terms of geometry, boundary conditions and time resolution of the problems. The results of this procedure are then exposed in Sec. 4 .

\section{Model description and validation}

\subsection{Initial model}

\subsubsection{Conservation equations}

The general form of the conservation equations for heat and moisture in porous building materials is briefly recalled here. It follows common notations and simplifying hypotheses used in the field of building physics [10, 13, 40]:

- local thermal and mass equilibrium between phases is assumed,

- air movement is not considered,

- thermodiffusion, hysteresis effects and chemical reactions are not considered,

- the temperature-dependency of the moisture storage is neglected.

\section{Moisture}

The mass conservation equation for water relates the temporal variations of the moisture content per unit volume $w\left[\mathrm{~kg} . \mathrm{m}^{-3}\right]$ to the moisture flow in either vapour or liquid phase, respectively denoted $\mathbf{g}_{v}$ and $\mathbf{g}_{l}$ :

$$
\frac{\partial w}{\partial p_{c}} \frac{\partial p_{c}}{\partial t}=-\nabla \cdot(\mathbf{g})=-\nabla \cdot\left(\mathbf{g}_{v}+\mathbf{g}_{l}\right)
$$

Under the aforementioned assumptions (air flow is neglected), water vapour transfer is only caused by diffusive phenomena, described by Fick's law and driven by a gradient of vapour pressure $p_{\nu}$. Liquid transport is driven by a gradient of capillary pressure $p_{c}$, according to Darcy's law.

$$
\begin{aligned}
\mathbf{g}_{v} & =-\delta_{p} \nabla p_{v} \\
\mathbf{g}_{l} & =-K_{l} \nabla p_{c}
\end{aligned}
$$

where $\delta_{p}$ and $K_{l}$ respectively denote the water vapour and liquid permeability of the material. In practical applications, only one pressure variable is used as a potential for moisture transfer. In order to guarantee the applicability of Eq. 1, the equivalence between $\nabla p_{v}$ and $\nabla p_{c}$ must be established. This equivalence can be formulated thanks to the Young-Laplace, Kelvin and Clausius-Clapeyron laws:

$$
\nabla p_{\nu}=\frac{p_{\nu}}{\rho_{l} R_{v} T} \nabla p_{c}+\frac{p_{v}}{\rho_{l} R_{v} T^{2}}\left[\rho_{l} L_{\nu}-p_{c}\right] \nabla T
$$

where $\rho_{l}, R_{\nu}$ and $L_{\nu}$ are the density, the specific mass constant and the latent heat of vaporisation of water, and $T$ is the temperature.

Using Eq. 1 for the prediction of moisture flow requires the knowledge of the material's equilibrium moisture content $w=f\left(p_{c}\right)$, as well as its unsaturated moisture permeability. Simulations performed in the present work involve five different materials, two of which have undergone hygric characterisation in previous papers [31, 29], while the properties of the three other materials have been extracted from the literature. The geometries of the simulation cases, along with the corresponding references for material properties, are defined in Sec. 3.1. 
Under the aforementioned hypotheses (no air transfer and no heat source term), the conservation equation for the total energy, expressed in terms of enthalpy, reads [40]:

$$
\frac{\partial \rho h}{\partial t}=-\nabla \cdot\left(\mathbf{q}_{c}+\mathbf{q}_{a}\right)
$$

The first term of this equation, the mixture enthalpy of the system (liquid water, humid air and solid material), is:

$$
\rho h=\left(\rho_{0} c_{0}+c_{l} w_{l}+c_{\nu} \rho_{\nu}\right)\left(T-T_{r e f}\right)+\rho_{\nu} L_{\nu}
$$

where $\rho_{0}$ and $c_{0}$ are the density and specific heat capacity of the dry material, $c_{l}$ and $c_{\nu}$ the heat capacities of liquid water and vapour, and $T_{r e f}=0^{\circ} \mathrm{C}$ the reference temperature. $w_{l}$ and $\rho_{\nu}$ respectively denote the liquid and gaseous parts of the total moisture content per unit volume $w=w_{l}+\rho_{\nu}$. Considering a constant value of the specific heat of each phase, and assuming that the gaseous phase does not significantly contribute to moisture nor heat storage $\left(\rho_{v} \ll w_{l}\right)$, the derivative of Eq. 6 reads:

$$
\frac{\partial \rho h}{\partial t}=\left(\rho_{0} c_{0}+c_{l} w_{l}\right) \frac{\partial T}{\partial t}+c_{l}\left(T-T_{r e f}\right) \frac{\partial w}{\partial t}
$$

In Eq. 5, $\mathbf{q}_{c}$ denotes the conductive heat transfer described by Fourier's law, and $\mathbf{q}_{a}$ denotes the advective transfer driven by moisture flow in both phases:

$$
\begin{aligned}
\mathbf{q}_{c} & =-\lambda \nabla T \\
\mathbf{q}_{a} & =c_{l}\left(T-T_{r e f}\right) \mathbf{g}+L_{\nu} \mathbf{g}_{v}
\end{aligned}
$$

The thermal conductivity $\lambda$ and effective capacity $c$ of the system are functions of the moisture content. Following these definitions, the second term of Eq. 5 can be developed as such:

$$
\begin{aligned}
-\nabla \cdot\left(\mathbf{q}_{c}+\mathbf{q}_{a}\right) & =-\nabla \cdot\left[-\lambda \nabla T+c_{l}\left(T-T_{r e f}\right) \mathbf{g}+L_{\nu} \mathbf{g}_{\nu}\right] \\
& =-\nabla \cdot\left[-\lambda \nabla T+L_{\nu} \mathbf{g}_{\nu}\right]-c_{l} \mathbf{g} \cdot \nabla T-c_{l}\left(T-T_{r e f}\right) \nabla \mathbf{g}
\end{aligned}
$$

which leaves two possibilities regarding the formulation of the heat balance equation: Eq. 1 can be integrated into Eq. 5 in order to cancel the last terms of Eq. 7 and 10.

$$
\begin{aligned}
\left(c_{0} \rho_{0}+c_{l} w\right) \frac{\partial T}{\partial t}+\left(c_{l} T \frac{\partial w}{\partial p_{c}}\right) \frac{\partial p_{c}}{\partial t} & =-\nabla \cdot\left[-\lambda \nabla T+c_{l} T \mathbf{g}+L_{\nu} \mathbf{g}_{\nu}\right] \\
\left(c_{0} \rho_{0}+c_{l} w\right) \frac{\partial T}{\partial t} & =-\nabla \cdot\left[-\lambda \nabla T+L_{\nu} \mathbf{g}_{\nu}\right]-c_{l} \mathbf{g} \cdot \nabla T
\end{aligned}
$$

Some authors prefer use Eq. 11 in order to keep the term of capillary pressure temporal derivative $\partial p_{c} / \partial t$ for a more straightforward spatial discretisation of the conservation equation [13]. Other authors [10] consider the sensible heat negligible in comparison to the term of latent heat flow $L_{\nu} \mathbf{g}_{v}$, which is equivalent to writing off the last term of Eq. 12.

\section{Boundary conditions}

The heat and moisture balance at an external surface is driven by the atmospheric boundary conditions. Fig. 1 sums up the notations used for their expression, which follow standardised building physics notations [10, 13].

The moisture exchange $g$ between the environment and the surface is driven by vapour exchange including evaporation and condensation, and by wind-driven rain impacting the facade:

$$
g=\beta\left(p_{v, a}-p_{\nu}\right)+g_{l, r}
$$




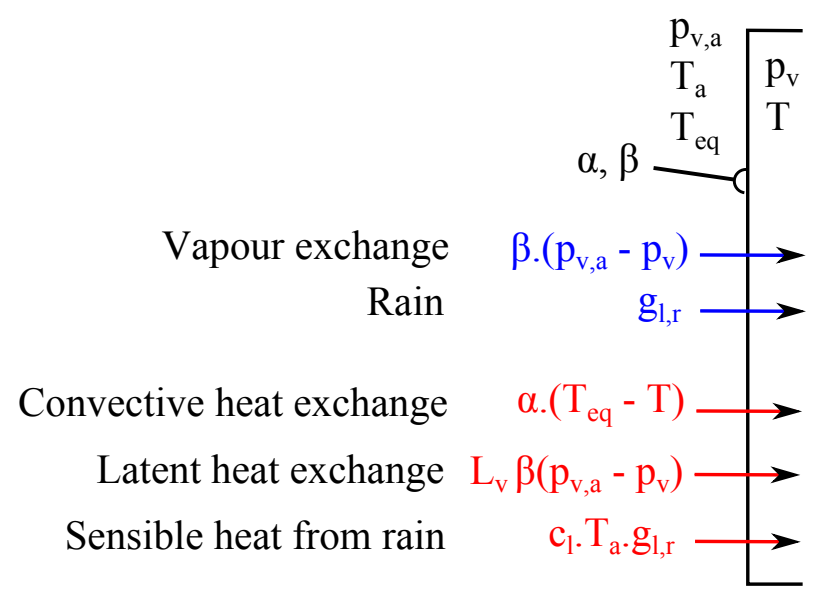

Figure 1: Expression of the boundary conditions

where $\beta$ denotes the surface moisture transfer coefficient, $p_{v, a}$ is the ambient vapour pressure and $g_{l, r}$ denotes an incoming liquid flow in the form of rain.

The heat flux $q$ from the environment to the surface includes a term of convective exchange, the sensible heat transfer due to precipitation, and the latent heat transfer due to vapour exchange:

$$
q=\alpha\left(T_{e q}-T\right)+c_{l}\left(T_{a}-T_{r e f}\right) g_{l, r}+L_{\nu} \beta\left(p_{\nu, a}-p_{\nu}\right)
$$

where $\alpha$ denotes the surface heat transfer coefficient and $T_{e q}$ denotes an equivalent external temperature, which combines the temperature of the ambient air $T_{a}$, along with the effects of short wave solar radiation and long wave ground radiation. The last term of Eq. 14 is written assuming that the moisture accumulation in the porous network mostly occurs in liquid form (either due to adsorption or to capillary condensation) and contributes entirely to the latent heat of vaporisation or condensation at the surface [10].

\subsubsection{Finite-element implementation}

The numerical implementation of Eq. 1 and 11 was performed with the finite-element method (FEM), mainly following the methodology presented by [13]. A simulation code was recently developed [31], and adapted so that experimental measurements of fracture geometries can be automatically integrated in a 2-dimensional FE mesh. The capillary pressure $p_{c}$ and the temperature $T$ were respectively chosen as driving potentials for moisture and heat flow.

The Galerkin weighted-residual method was used for the spatial discretisation over a triangular mesh of Lagrangetype quadratic elements. The temporal discretisation follows the first-order implicit scheme. As the storage and transport coefficients of the equations are functions of the field variables $p_{c}$ and $T$, the discretised system is strongly non-linear: the solution of each time step is approached iteratively, and a Newton-Raphson iterative scheme was used as to accelerate the convergence. Finally, a dynamic time stepping was implemented, adapting the size of each time step $\Delta t^{i+1}$ according to the number of iterations of the previous step $\Delta t^{i}$ :

$$
\Delta t^{i+1}=\min \left[\Delta t^{i} \min \left(\frac{m_{\max }}{2 m}, 2\right), \Delta t_{\max }\right]
$$

where $m_{\max }=12$ is the maximal authorised number of iterations per time step. Should convergence not be reached at the end of this series of iterations, a new attempt is made with half the size of the time step. Such a reduction for instance occurs at the beginning of rain showers, decreasing the time step to the order of seconds. The step then increases when climatic conditions allow a faster convergence, and is bounded by a maximal value of $t_{\max }=10 \mathrm{~min}$ as to not exceed the climatic input frequency.

The presented methodology consists of long-term simulations including highly variable boundary conditions such as sudden rain showers. Such an adaptative time stepping procedure allows finding a compromise, in order to 
account for all variations of the climatic data while avoiding tremendous calculation times. Previous questionings on the required time resolution for the wind and rain input data in order to obtain accurate calculation results show that time steps of up to 10 min yielded a good agreement with experimental data [4]. Hourly or daily time resolutions based on an arithmetic averaging of the climatic data can however imply important errors: these errors may be reduced by using a weighted averaging technique [5].

\subsubsection{Validation}

The Hamstad project was initiated as to develop a platform for the assessment of computational HAM modelling in building physics. The first package of the project [24] addressed material characterisation, while the second package [12] established a standard methodology of HAM modelling, in order to provide an alternative to the Glaser method for calculation, prediction and evaluation of the moisture performance of the building enveloppe. As part of this work package, a series of benchmarks were proposed [11] in order to cover various combinations of climatic loads and of materials, and to deal with resulting phenomena: internal condensation, moisture movement caused by thermal gradients, effect of water on the thermal conductivity...

The $4^{\text {th }}$ benchmark exercise is used here to illustrate the validity of the presented model in comparison to other existing codes. It deals with the one-dimensional moisture movement inside a wall with a hygroscopic finish. The simulation time is 5 days, during which highly changing climatic conditions are imposed, successively generating moisture condensation, redistribution and evaporation. The complete description of the exercise and its components was given by Hagentoft et al [10, 11]. Tariku et al. [40] recently used it for model validation as well.

The boundary conditions are prescribed in the form of heat and moisture flows, given in Eq. 13 and 14. Hourly values of the external and internal air temperature and vapour pressure are given, as well as an equivalent external temperature $T_{e q}$ including the influence of solar radiation, and the liquid inflow caused by rain $\mathbf{g}_{l, r}$. The case is complicated by capillary active materials of very high moisture permeability. The thermal conductivity and hygric parameters of such materials can be strongly influenced by the moisture content.

Moisture transfer is a strongly non-linear phenomenon and the resolution of Eq. 1 and 11 involves important mathematical difficulties. The considered benchmark case being particularly demanding, the numerical results obtained by the participants may differ, although the simulation codes apply the same transport equations. A statistical approach is used for the evaluation of the solution, based on the analogy with a $t$-distribution. A band of acceptance is defined as the range within which results are considered acceptable:

$$
\bar{x}-t_{p} \frac{\sigma_{x}}{\sqrt{n}} \leq \mu \leq \bar{x}+t_{p} \frac{\sigma_{x}}{\sqrt{n}}
$$

where $\bar{x}$ and $\sigma_{x}$ are the main value and standard deviation of the sample set, $n$ is the number of observations and $t_{p}$ is a function of $n$ and the confidence grade $p$, defined as the risk the band of acceptance does not contain the true numerical solution. For a given value of $p$, an increase of the number of participants reduces $t_{p}$ and narrows the band of acceptance, as the probability of knowing the real solution of the problem increases. In the following display of the results, the confidence interval was set to $p=1 \%$, i.e. a $99 \%$ chance that the band contains the correct solution, resulting in a value of $t_{p}=3.71$.

The results obtained by the six participants of the benchmark calculations were compared in terms of temporal evolution of the field variables at given locations in the wall, and of spatial distributions at given times of simulation. The results of the presented model are shown on Fig. 2 and 3, respectively depicting the evolution of the moisture content and of the temperature, at the outer surface of the facade, during the five days of simulation.

On each graph, the red continuous line shows the distribution obtained by the current model, while the dotted lines show the average distribution of the other six participants, as well as the limits of the $99 \%$ confidence interval. During the entire time of simulation, the external air temperature and vapour pressure, and the internal equivalent temperature, are constant. The variations of the other boundary conditions roughly occur in three main successive stages.

- The first phase lasts from 0 to 49 hours of simulations: a drop of the equivalent external temperature to $-2^{\circ} \mathrm{C}$ generates condensation on the external wall surface, and the moisture content of the internal layer rises due to an increase of internal vapour pressure. 


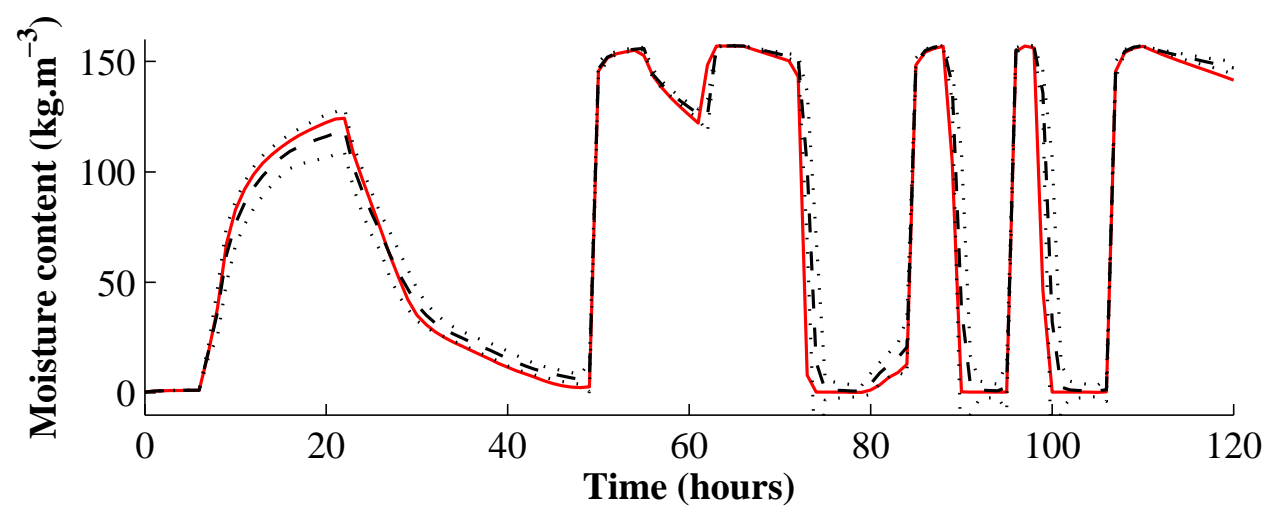

Figure 2: Moisture content at the outer surface

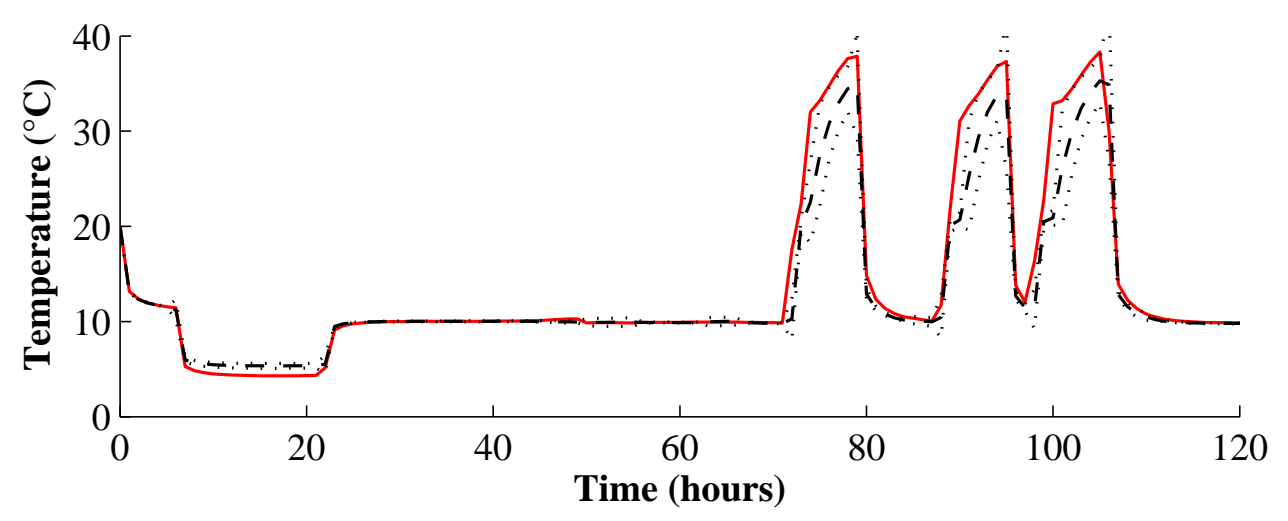

Figure 3: Temperature at the outer surface

- The second phase lasts from 50 to 66 hours: all temperatures are constant, and two closely successive rain showers occur. The first rain shower generates a sudden rise of the external surface moisture content to the state of saturation. After the rain stops, this concentration decreases as the material dries, and because of moisture redistribution. Shortly after the second rain shower, the moisture front reaches the second material layer (hygroscopic finish) and the moisture content of the internal surface rises as well.

- The third phase lasts from 67 to 120 hours. Square-wave fluctuations of the external equivalent temperature between 10 and $50^{\circ} \mathrm{C}$ occur alternating with rain showers. Because of the high mositure diffusivity of the load bearing material, these rain showers prevent the internal layer from drying. Temperature variations quickly affect the external surface temperature, and affects the internal surface temperature with some delay.

The performance of the proposed model during these solicitations offers a fair accordance with the statistical analysis based on the previous results of the other participants. The calculated moisture content distributions mostly stay within the band of acceptance. However, the evolution of the external and internal surface temperature diverges slightly from the expected values when the ambient thermal load is important. This implies a light overestimation of the moisture condensation at the internal layer during the first phase of the simulation protocol. This stage of the simulation is particularly sensitive to the expression of the coupling between heat and moisture transfer, particularly to the value of the latent heat of evaporation $L_{v}$. Despite these light deviations, the simulation code can be considered appropriately able to predict coupled heat and moisture transfer in multi-layered facades, under heavy climatic loads, during longer times of simulation. 


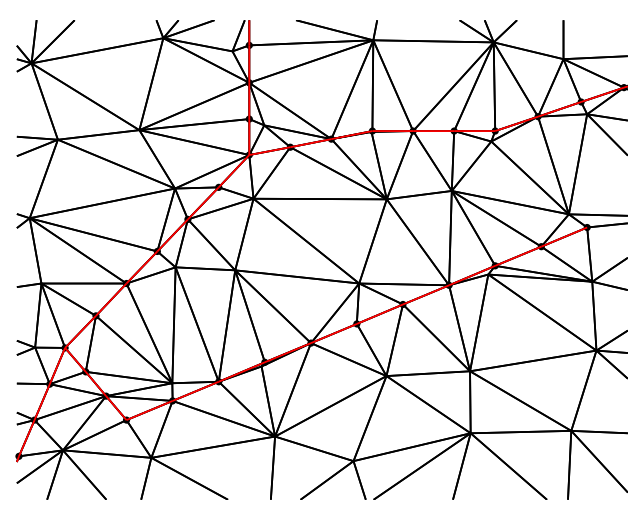

Figure 4: Adaptation of the FE mesh to the fracture geometries

\subsection{Flow in fractured porous media}

\subsubsection{Fracture integration}

The numerical model for heat and moisture transfer has been validated in regards to isotropic porous media. In order to meet the target of the study, it must now be extended as to allow including the effects of fractures on the flow. Fracture positions and sizes can either come from a manual input or from experimental measurements: in a previous work, digital image correlation was used during the mechanical loading of concrete samples. This technique provides two-dimensional displacement mappings, from which crack networks could be derived following an appropriate image processing methodology [28]. The result of this procedure is the quantification of all visible cracks in the form of a distributed set of segments of known aperture.

The present numerical study, as well as the previous experimental work, is limited to a 2D observation of flow in fractured porous media, as it was considered sufficient to support the point of the paper. A more comprehensive account of cracks would however require a 3D description in order to include all their geometrical parameters. The procedure for the integration of such fracture segments into a FE mesh is as follows: an initial 2D unstructured triangular mesh is generated over the geometry of the considered material layer. Nodes are added along the fracture segments, discretising each one into smaller elements. The initial mesh is then readjusted by Delaunay triangulation based on all available nodes, so that fracture segments match element edges. An example is shown on Fig. 4.

The present model therefore belongs to the category of mesh-adapting models. Cracks are explicitly modelled in the primary FE mesh, as opposed to staggered approaches [25, 2, 19, 23] in which transport equations are solved separately in the fractures and in the porous medium. For such a fully coupled analysis, fracture elements can be double-nodded for a full account of transverse diffusion mechanisms through the interfaces [35]. A less comprehensive formulation is however privileged here for simplification purposes. Another potential of improvement of the current methodology is the fact that the current mesh is not optimised near the discontinuities, resulting in possible stretched element shapes (see Fig. 4). This can be improved by envisaging more advanced refinement strategies [32,33].

Once integrated into the FE mesh, specific transport properties are attributed to fracture nodes. This matter is illustrated on Fig. 5. The expression of the liquid water permeability $K_{l}$ of these nodes is replaced by a permeabiliy tensor $\mathbf{k}_{f}$ such as:

$$
\mathbf{k}_{f}=\mathbf{R}^{T}\left(\begin{array}{cc}
k_{f, l} & 0 \\
0 & k_{f, t}
\end{array}\right) \mathbf{R}
$$

in which $k_{f, l}$ and $k_{f, t}$ are the respective values of the longitudinal and transverse fracture permeability, and $\mathbf{R}$ is the rotation matrix for directing the permeability tensor in the direction of the crack. The expression of the 

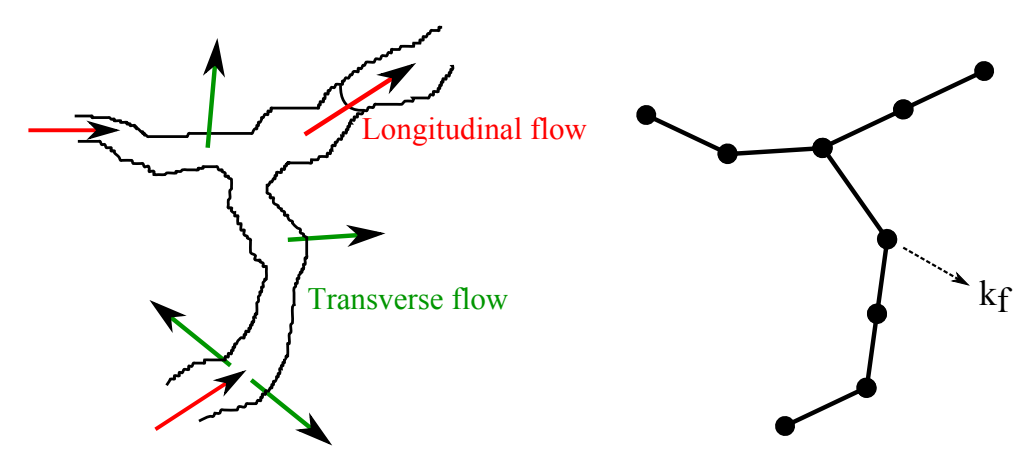

Figure 5: Fracture discretisation and expression of the longitudinal and transverse flow

longitudinal permeability in a water-saturated crack of aperture $u$ results from the analytical solution of the NavierStokes equations for flow between two parallel plates [38]:

$$
k_{f, l}=\frac{\rho_{l}}{\eta_{l}} \frac{u^{2}}{12} \quad \text { if } \quad\left|p_{c}\right| \leq \frac{2 \sigma}{u}
$$

This saturated permeability applies if the value of the capillary pressure meets an occupancy criterion proposed by [41] and derivated from the Young-Laplace equation. Higher values of $\left|p_{c}\right|$ imply that the fracture segment is not filled with water, and this expression is no longer valid. For simplification purposes, the non-saturated longitudinal fracture permeability was set so as to reach a computational value of zero (namely $10^{-17} \mathrm{~s}$ ) at dry conditions $\left(\left|p_{c}\right|=10^{9} \mathrm{~Pa}\right)$. This is shown on Fig. 6 with the example of a crack of aperture $u=2 \times 10^{-6} \mathrm{~m}$.

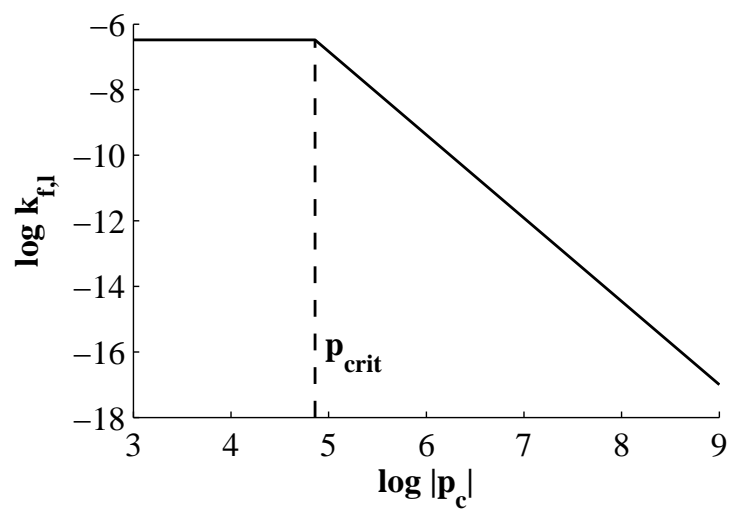

Figure 6: Fracture discretisation and expression of the longitudinal and transverse flow

Transverse flow in the cracks, as well as effects of the resistance of the crack/matrix interfaces to moisture flow, were neglected: this involves that the transverse permeability $k_{f, t}$ written in the $\mathbf{k}_{f}$ tensor was set to the same value as in the surrounding porous medium.

This procedure, although it does not encompass an extensive description of flow mechanisms at the smaller scales, is considered satisfactory for its accurate description of the real fracture geometries and its relatively simple implementation. Moreover, the concept can be extended as to include the effects of the longitudinal and transverse thermal conductivities of fractures. Further possibilities for the improvement of the code include:

- a more comprehensive description of the unsaturated permeability and storage capacity of fractures segments as function of their aperture and rugosity [41],

- the optimisation of the mesh refinement strategy near the integrated fractures [32], 
- the integration of the transverse fracture flow [35].

The aforementioned simplifications are expected to have little impact on the simulation results, but may influence the overall performance of the code. This will be discussed after the display of the results.

\subsubsection{Validation}

Prior to running the simulations required by the objectives of the study, the model must be validated in regards to two criteria. The first one was met in Sec. 2.1.3, where it was showed that the behaviour of multi-layered walls under heavy climatic loads could be accurately predicted. The second requirement is to show that the effect of fractures on moisture flow is properly accounted for by the presented model.

Two aspects must be considered when evaluating the accuracy of the calculations: the prediction of water infiltration in the fractures and of the overall fluid flow in the porous medium resulting from their presence. This latter concern was addressed in a previous study [31], in which a first version of the simulation code was developed and tested: two-dimensional moisture content distributions were measured by X-ray radiography in cracked concrete samples, and the simulation code was used to recreate these distributions based on the knowledge of the crack geometry, measured by digital image correlation. Fracture flow was not calculated, as cracks were considered initially water-filled: this assumption coincides with the observation that water infiltration in cracks occurs in a much smaller time frame than in the porous medium. Predictions of two-dimensional moisture content distributions however showed a fair accordance with longer-term measurements, several minutes after wetting. Additional tests were carried and displayed in a further study [30]. The simulation code was then extended as to explicitly calculate water infiltration and drainage in fractures, as described above. This aspect has however not been subjected to experimental validation, as it has little impact on long-term simulations: its purpose is mostly to account for the reversibility of water infiltration in fractures [27].

\section{Simulation settings}

The target of the study is the application of the validated model to the evaluation of the hygrothermal performance of building components integrating fractured material layers. For this purpose, a series of simulations has been performed, so that the comparison of their results gives an estimate of how fractures may influence phenomena such as moisture accumulation and heat loss.

These simulation cases have been defined in order to answer a threefold questioning:

- to estimate the consequences of fractures on the moisture accumulation and heat loss of a standard wall,

- to find whether these consequences are aggravated by the infiltration of moisture towards insulation materials,

- to illustrate how outer insulation including hygrophobic materials can correct the flaws generated by fractures.

\subsection{Geometry}

\subsubsection{Components and materials}

The setup of the simulated walls and the list of the corresponding materials, are shown on Fig. 7. Three facades have been defined: a simple non-insulated wall with an internal finishing layer (case 1), a similar wall including inner insulation (case 2) and one including outer insulation (case 3). In all cases, the load-bearing material is a layer of concrete of $20 \mathrm{~cm}$, and a gypsum board of $1.25 \mathrm{~cm}$ thickness is added at the internal surface. Case 2 includes a $10 \mathrm{~cm}$ layer of interior insulation (fibreboard), and case 3 includes an outer insulation made of $1 \mathrm{~cm}$ hygrophobic coating (fibre-reinforced mortar) and $10 \mathrm{~cm}$ insulation (mineral wool). The thermal and hygric properties of these materials are summed up in Tab. 1. 


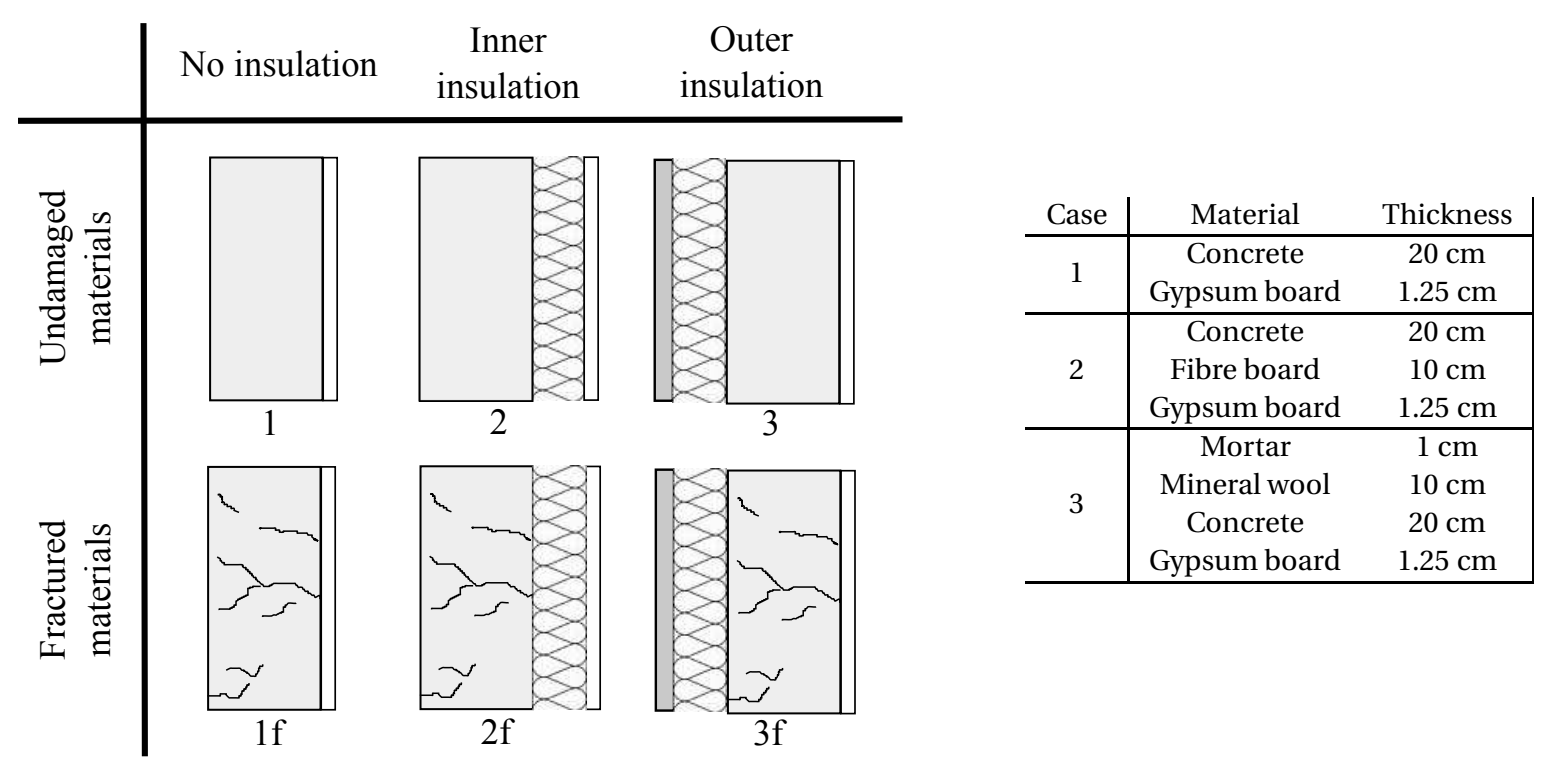

Figure 7: Definition of the simulation cases and materials

The moisture transport and storage properties of concrete and mortar have been previously characterised $[31,29]$. Their thermal properties, were taken from databases or from the literature concerning similar materials [15]. All thermal and hygric properties of the gypsum board are those of the finishing material defined in the $4^{\text {th }}$ benchmark of the Hamstad package [11], while the fibre board is the capillary active insulation of the $5^{\text {th }}$ benchmark. The exterior insulation includes a formulation of mineral wool, of which properties were taken from [14]. The thermal conductivity of all materials is a linear function of the moisture content. Their sorption isotherm $w$ (equilibrium moisture content) is approached by a multimodel van Genuchten model, neglecting hysteresis effects, and the vapour permeability $\delta_{p}$ by a Schirmer model. The expression of the liquid permeability differs between each material : multi-modal Durner model for concrete [31], exponential approximation for the gypsum and fibre boards [11].

To each simulation case is assigned an equivalent wall, in which the concrete layer integrates a network of fractures. These fractures have been generated in the frame of a previous study by an interrupted tensile loading of fibre reinforced concrete samples [31]. Although this specific geometry is not expected to be representative of all crack configurations which may arise in real operating conditions, it has been considered fit for justifying the presented methodology.

\subsubsection{Mesh}

The most mathematically challenging phenomenon occuring in the presented simulations is moisture transfer in the liquid phase, which generates steep gradients of capillary pressure and of the related transport and storage coefficients. More specifically, the abruptness of the permeability profile, and of the retention curve, is key: the mesh density of a material must be high enough to ensure a sufficient smoothness of their local variations. A multilayered wall therefore consists of several concatenated mesh densities. The fracture segments have the highest saturated moisture permeability, and are discretised with intervals of $3 \times 10^{-4} \mathrm{~m}$. A non-structured triangular mesh is applied on the surrounding porous medium, integrating the crack geometry. The distance between nodes is then set according the each material's moisture permeability, and ranges from $1 \mathrm{~mm}$ for gypsum board to $3 \mathrm{~mm}$ for concrete. In simulating the undamaged walls (case 1, 2 and 3), a quasi-1D mesh was generated with a narrow rectangular geometry and two insulation boundary conditions. The walls including a fractured concrete layer (case 1f, $2 \mathrm{f}$ and $3 \mathrm{f}$ ) were modelled in $2 \mathrm{D}$ with a height of $20 \mathrm{~cm}$. As an illustration, the mesh used for the simulation case If is displayed on Fig. 8.

Although the current settings of the meshing procedure allow including any fracture geometry into the finite- 


\begin{tabular}{|c|c|c|c|c|c|c|}
\hline & & $\begin{array}{c}\text { Concrete } \\
{[31]}\end{array}$ & $\begin{array}{c}\text { Gypsum } \\
{[11,15]}\end{array}$ & $\begin{array}{c}\text { Fibre board } \\
\quad[11]\end{array}$ & $\begin{array}{c}\text { Mortar } \\
{[29]}\end{array}$ & $\begin{array}{c}\text { Mineral wool } \\
{[14]}\end{array}$ \\
\hline \multirow{2}{*}{ Heat capacity $c_{0} \rho_{0}$} & $c_{0}\left(\mathrm{~J}^{\mathrm{kg}} \mathrm{kg}^{-1}\right)$ & 880 & 870 & 1000 & 920 & 800 \\
\hline & $\rho_{0}\left(\mathrm{~kg} \cdot \mathrm{m}^{-3}\right)$ & 2450 & 790 & 212 & 230 & 170 \\
\hline \multirow{2}{*}{$\begin{array}{l}\text { Thermal conductivity } \lambda \\
\lambda_{0}+\lambda_{w} \frac{w}{\rho_{l}}\end{array}$} & $\lambda_{0}$ & 1.75 & 0.2 & 0.06 & 0.6 & 0.047 \\
\hline & $\lambda_{w}$ & 0.45 & 0.45 & 0.56 & 0.45 & 0.7237 \\
\hline \multirow[t]{2}{*}{ Equilibrium moisture content $w$} & $w_{\text {sat }}\left(\mathrm{kg} \cdot \mathrm{m}^{-3}\right)$ & 101.44 & 209 & 871 & 200 & 209.6 \\
\hline & $l$ & 1 & 1 & {$[0.41 ; 0.59]$} & {$[0.2 ; 0.8]$} & {$[0.04 ; 0.96]$} \\
\hline \multirow{2}{*}{$w_{s a t} \sum_{i} l_{i}\left[1+\left(\alpha_{i}\left|p_{c}\right|\right)^{\frac{1}{1-m_{i}}}\right]^{-m_{i}}$} & $\alpha\left(\times 10^{-6} \mathrm{~Pa}^{-1}\right)$ & 0.62 & 2.0 & {$[0.6 ; 1.2]$} & {$[51 ; 0.4]$} & {$[0.5 ; 10]$} \\
\hline & $m$ & 0.22 & 0.21 & {$[0.60 ; 0.58]$} & {$[0.33 ; 0.74]$} & {$[0.3 ; 0.7]$} \\
\hline Vapour permeability $\delta_{p}$ & $\mu$ & 30 & 3 & 5.6 & 50 & 5 \\
\hline$\frac{26.1 \times 10^{-6}}{\mu R_{\nu} T} \frac{1-w / w_{\text {sat }}}{(1-p)\left(1-w / w_{\text {sat }}\right)+p}$ & $p$ & 0.5 & 0.5 & 0.2 & 0.2 & 0.5 \\
\hline \multicolumn{2}{|l|}{ Liquid permeability $K_{l}$} & [31] & BM 4 [11] & BM 5 [11] & 0 & [14] \\
\hline
\end{tabular}

Table 1: Thermal and hygric properties of the materials

element mesh and predicting flow accordingly, some improvements remain to be made for its optimisation (see Sec. 2.2). For instance, the current algorithm does not allow a progressive increase of the mesh density near fractures: the mesh is therefore fully refined in the entire material layer that includes fracture segments. This involves high mesh densities in locations where they are not required, and consequently increases the computational time. The current code also does not allow simultaneously using triangular and square-shaped finite-elements: a triangular non-structured mesh is applied on the non-fractured material layers as well.

\subsection{Boundary conditions}

In the present work, the exterior boundary conditions of the problem are implemented according to climatic data files measured in Lyon (France) during the year 2011. The following quantities are available as instantaneous measurements recorded with time steps of one minute: altitude and azimuth of the sun, diffuse and direct horizontal irradiance, wind speed and direction, relative humidity, horizontal rainfall intensity and dry bulb temperature.

Prior to the calculations, these parameters have been translated into the variables shown in Eq. 13 and 14 as to be included in the simulation code. First, the relative humidity and air temperature can be directly used in the expression of the boundary heat and moisture flows. Then, the convective transfer coefficients $\alpha$ and $\beta$ are functions of the local air velocity on the building surface, determined by its orientation and by the wind speed and direction [13]. The effects of solar radiation are then integrated in the expression of an equivalent temperature $T_{e q}$ at which the heat transfer rate due to the temperature across the wall is the same as the rate due to the combined effects of convection, conduction and radiation:

$$
\alpha\left(T_{e q}-T\right)=\alpha\left(T_{a}-T\right)+\kappa\left(I_{d i r}+I_{d i f}\right)
$$

where $I_{d i r}$ and $I_{d i f}$ denote the direct and diffuse solar irradiance, and $\kappa$ is the absorptivity of the exposed material. This value was set to that of concrete and mortar $\kappa=0.6$, as these materials form the external layers of the simulated walls. Long-wave radiative exchange between the building surface and its environment is neglected. Wind-driven rain $\mathbf{g}_{l, r}$ is derived from the horizontal rainfall $R_{h}$ and the wind speed $U$ and direction, using a simplified empirical model [4] :

$$
\mathbf{g}_{l, r}=0.222 U R_{h}^{8 / 9}
$$

This expression does not explicitely account for the raindrop size distribution, nor does it include the impact of the building height and of the wind speed, but its description of the rain loads is considered appropriate for the current study. 


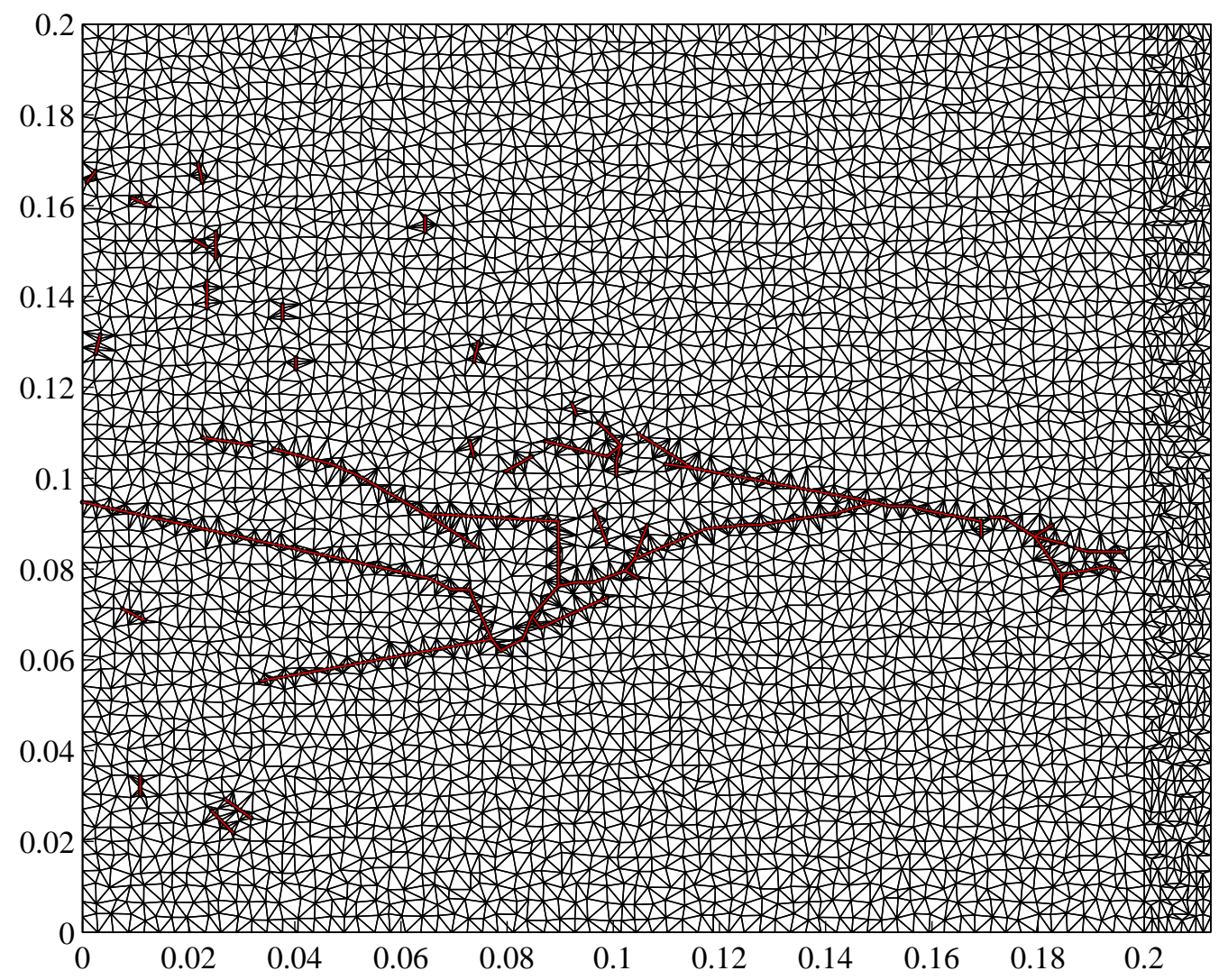

Figure 8: Generated mesh for the simulation case if

Climatic data were averaged over time steps of 10 minutes, although the calculation may proceed to smaller time steps in case of convergence difficulties. The boundary transfer coefficients, local air velocity at the external surface and the value of the direct solar irradiance were derived for the case of a westward facade. Furthermore, the hygrothermal behaviour of the walls was only observed during two separate months of simulations instead of an entire year: a winter month (february, see Fig. 9) and a summer month (july). Indeed, a full simulation year of fractured components would imply long computational times with the current settings of the model, and the two selected months are representative of the conditions to which the walls may be subjected.

Fig. 9 shows the boundary conditions imposed at the external surface of the wall configurations during the winter month. Due to the influence of solar irradiation, the equivalent temperature displays important variations and values exceeding the temperature of the air. The amount of rain impacting the facade shows peaks as rain showers coincide with wind directions leading water towards the facade: three such major rain events are visible during this month, along with several minor ones.

For simplification purposes, the interior boundary conditions of all simulation cases are constant: $\mathrm{RH}=75 \%$, $T=20^{\circ} \mathrm{C}, \alpha=7 \mathrm{~W} \cdot \mathrm{m}^{-2} \cdot \mathrm{K}^{-1}, \beta=3 \times 10^{-8} \mathrm{~s} . \mathrm{m}^{-1}$. Although a large part of the moisture income towards building materials originates from the indoor humidity level, influenced by the occupants, this matter was simplified here.

\section{Results}

The previous section has set the frame for this last step of the numerical work. The questioning of this numerical study was laid in the form of three targets, the first one of which is to estimate the consequences of fractures on 


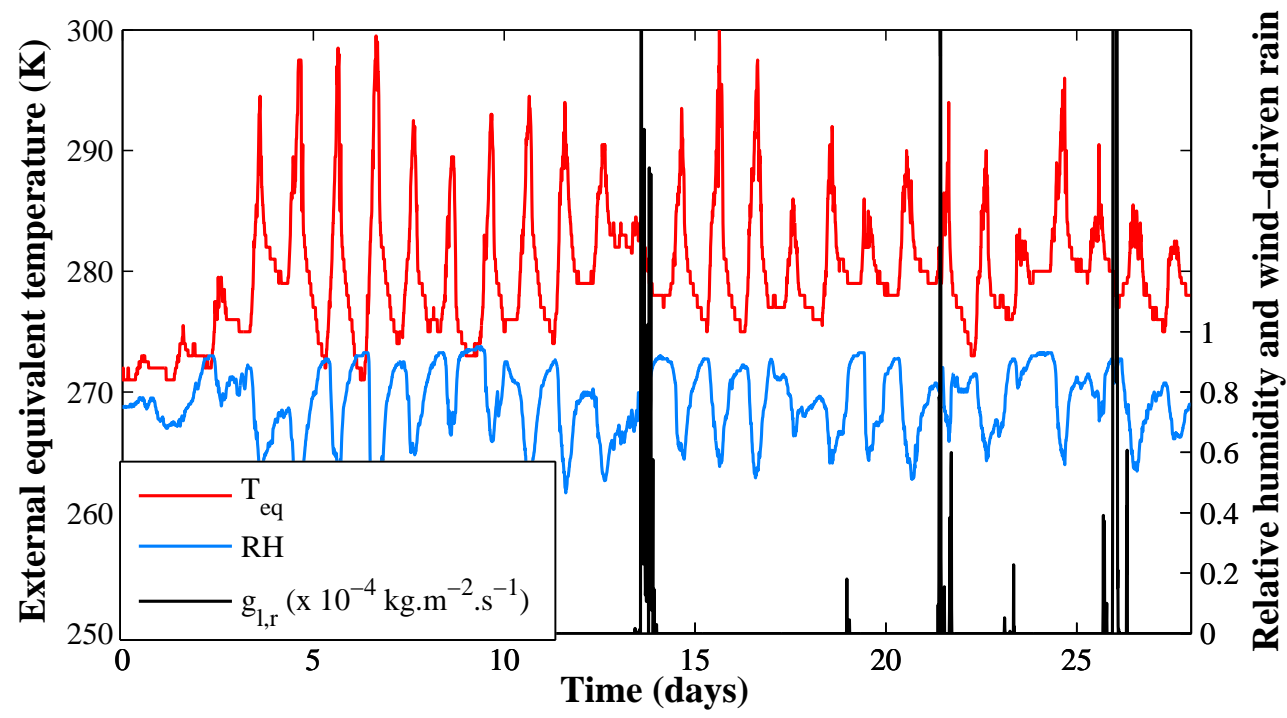

Figure 9: External equivalent temperature, relative humidity and wind-driven rain on a western facade during the month of february

the moisture accumulation and heat loss of a standard wall, by comparing the results of simulation cases 1 and lf. The second question is to find whether these consequences are aggravated by the infiltration of moisture towards insulation materials: this is done by comparing these two cases with cases 2 and $2 \mathrm{f}$. The last target is to illustrate how outer insulation may correct the flaws generated by fissures: it is the purpose of the cases 3 and $3 f$. The setup of the simulations, summarising the notations, wall geometry and boundary conditions, is illustrated on Fig. 10, which shows the output quantities defined to answer this questioning.

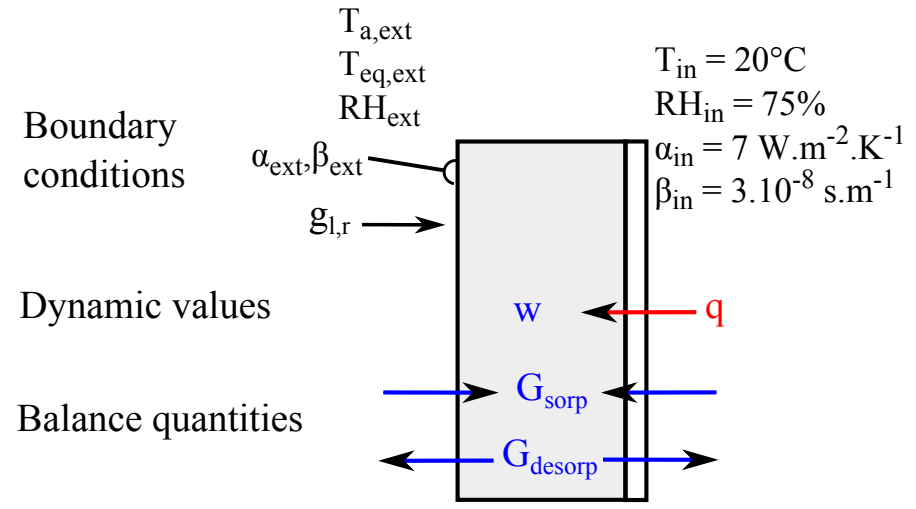

Figure 10: Simulation setup definition of the output notations

The results presented in the following section are twofold. In Sec. 4.1, temporal distributions are first showed for a qualitative illustration of the differences between several simulation cases. They are graphs showing the evolution, during a month of simulation time, of an instantaneous value. These values, illustrated on Fig. 10, are the heat flux towards the inner surface $\mathbf{q}\left[\mathrm{W} . \mathrm{m}^{-2}\right]$, and the average moisture content of each material layer $w_{m}$ $\left[\mathrm{kg} . \mathrm{m}^{-3}\right]$. The convention for quantifying the heat flow was chosen in order for $\mathbf{q}$ to be representative of the heat loss or gain of the inner environment.

The second class of variables used for the display of the results are integrated values used for quantitative 
comparisons in Sec. 4.2. $G_{\text {sorp }}$ and $G_{\text {desorp }}\left[\mathrm{kg} \cdot \mathrm{m}^{-2}\right]$ are respectively defined as the total raw intake and release of water between the wall and both inner and outer environments. They are calculated by integrating the positive or negative part of $g$ on both the external and the internal surfaces $S_{\text {ext }}$ and $S_{\text {int }}$ :

$$
\begin{aligned}
G_{\text {sorp }} & =\int_{t}\left[\int_{S_{\text {int }}+S_{\text {ext }}} \max (\mathbf{g}, 0) \mathrm{d} S\right] \mathrm{d} t \\
G_{\text {desorp }} & =-\int_{t}\left[\int_{S_{\text {int }}+S_{\text {ext }}} \min (\mathbf{g}, 0) \mathrm{d} S\right] \mathrm{d} t
\end{aligned}
$$

High values of both these quantities denote important moisture content fluctuations, while an high difference between them denotes an accumulation of moisture in the wall during the considered period. As both phenomena influence the material degradation, $G_{\text {sorp }}$ and $G_{\text {desorp }}$ can be used as indicators of the durability of a building component.

\subsection{Dynamic behaviour}

First, the dynamic behaviour of the walls is discussed. Each of the following graphs displays the temporal evolution of $w$ and $\mathbf{q}$ for both undamaged and damaged versions of one wall geometry, during one month of simulation. Fig. 11 and 12 show the behaviour of the non-insulated wall (cases 1 and 1f), while Fig. 13 and 14 show the behaviour of the inner insulated wall (cases 2 and 2f), respectively during the winter and the summer month. The initial conditions of the winter simulations are $T=10^{\circ} \mathrm{C}$ and $\mathrm{RH}=75 \%$, and those of the summer simulations are $T=20^{\circ} \mathrm{C}$ and $\mathrm{RH}=75 \%$. On each graph, the non-damaged wall is shown by the continuous lines, while the wall including a fractured concrete layer is shown by the dotted lines.

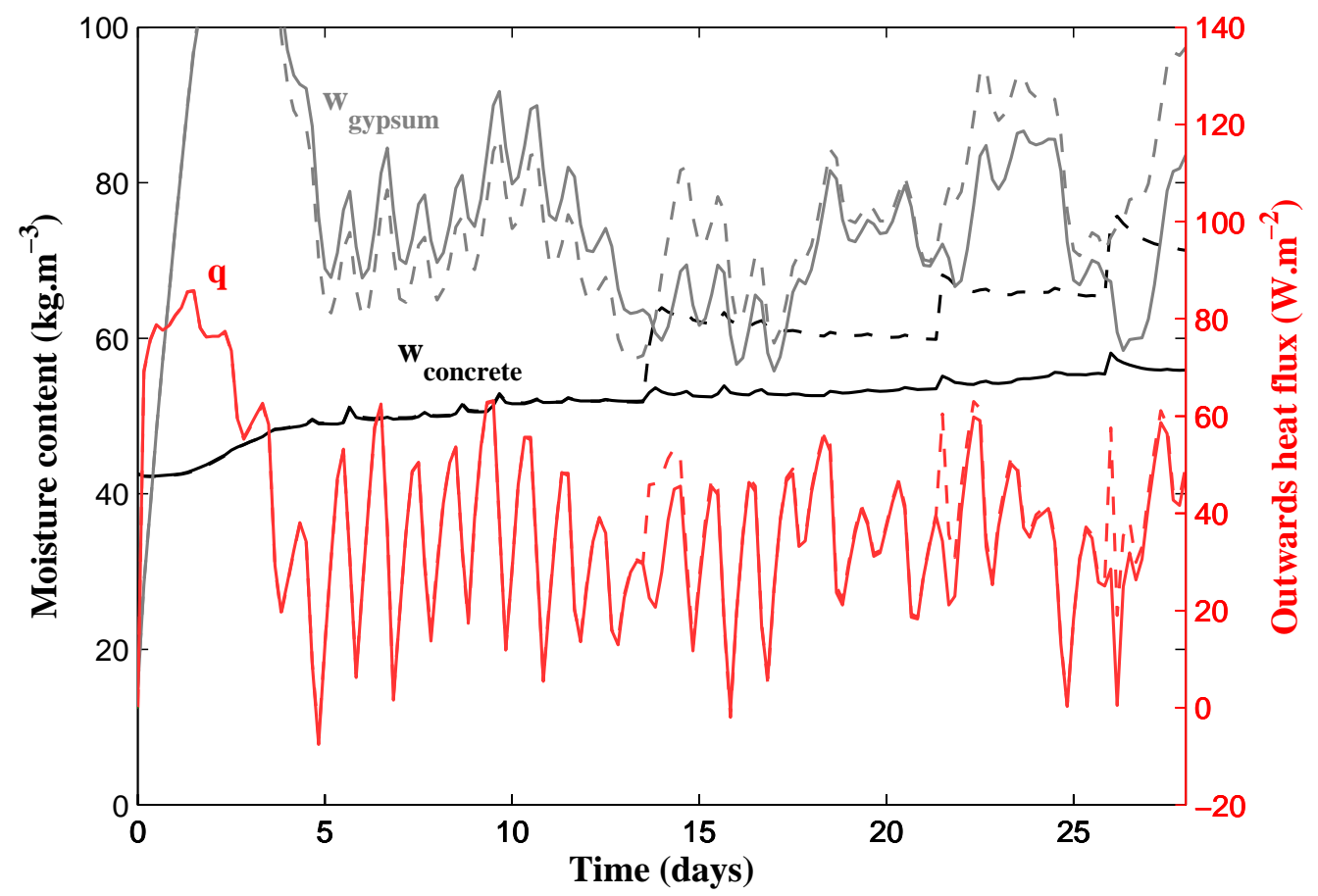

Figure 11: Non-insulated wall (february). Continuous lines: initial wall; dotted lines: fractured wall

The interpretation of Fig. 11 leads to the following observations: 
- The evolution of the concrete moisture content $w_{\text {concrete }}$ and of the heat loss $\mathbf{q}$ is almost identical during the first period of the simulations, whether the concrete layer is fractured or not. During this phase, moisture transfer only occurs in the gaseous phase and by adsorption of water vapour in the porous media. Cracks exposed to the ambient air have therefore little impact on the moisture content of the facade, and on its thermal properties, as they do not significantly accelerate moisture ingress in the vapour phase.

- A rain shower then occurs around $t=14$ days (see Fig. 9), causing an abrupt rise in the moisture content of the fractured concrete layer $w_{\text {concrete }}$ (black lines) due to water suction in the main fracture. The value of $w_{\text {concrete }}$ then slowly decreases during the drying of the facade, though an important accumulation of moisture remains in this material of low permeability. In the present case, this accumulation is aggravated by frequent rain showers preventing the complete drying of the wall.

- In the presence of fractures, some water migrates through the concrete layer and reaches the gypsum board at the internal surface: its moisture content $w_{\text {gypsum }}$ (gray lines) indeed shows increases after rain showers.

- The heat exchange $\mathbf{q}$ between the indoor air and the wall is displayed by the red lines on Fig 11. The only observable differences between the intact and the damaged walls in terms of thermal behaviour (continuous and dotted lines, respectively) occur shortly after rain showers. The heat flow from the inside is briefly increased in the presence of cracks: this is caused by a slight cooling of the wall due to water evaporation towards the external air, which is accentuated in the fractured case.

These results illustrate the potential impact of cracks on the hygric and thermal performance of a non-insulated wall. In this case, the main concern is the moisture accumulation inside the load-bearing material, which may significantly affect its durability. Fractures however seem to have very little impact on the thermal performance of this particular configuration.

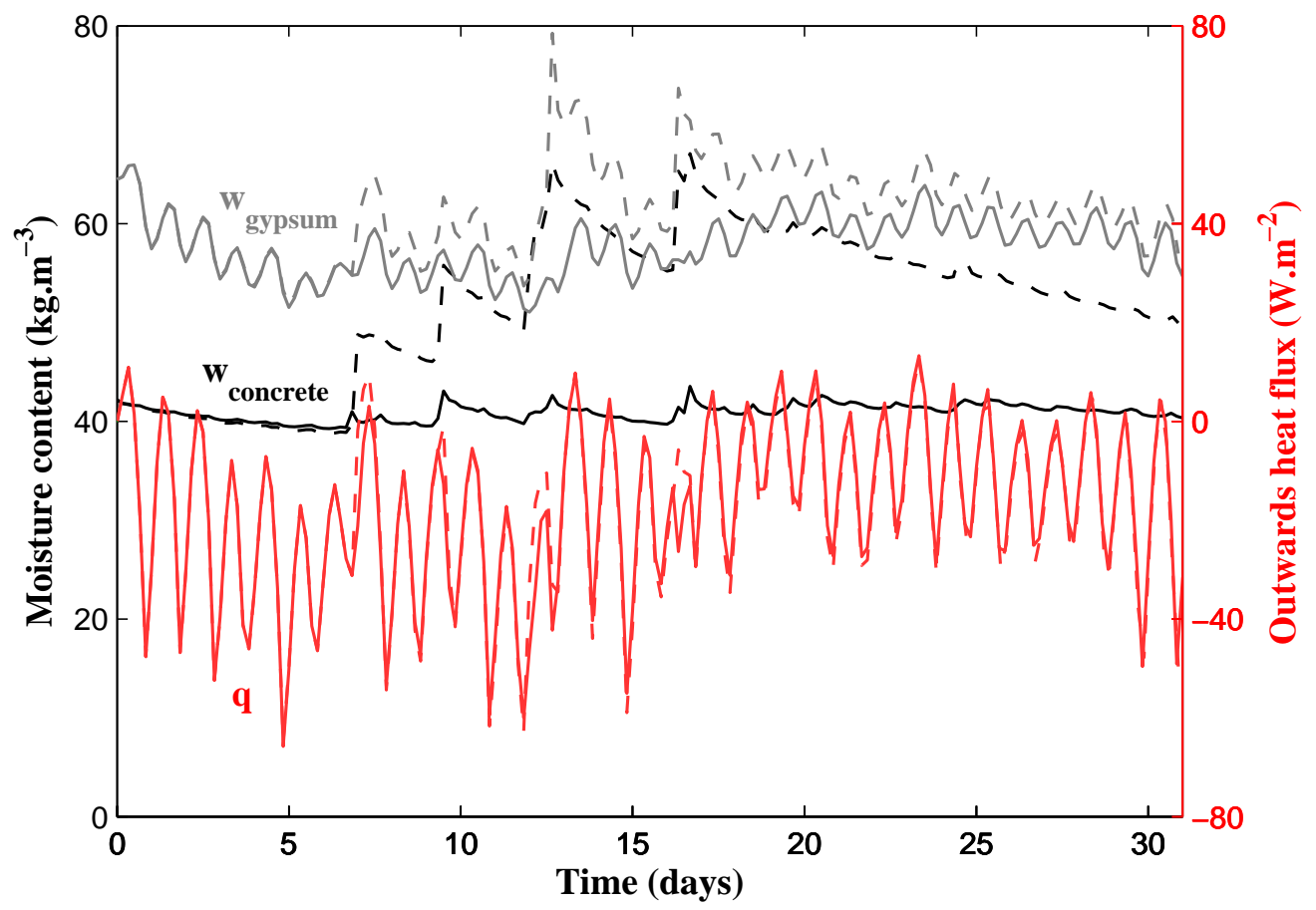

Figure 12: Non-insulated wall (july). Continuous lines: initial wall; dotted lines: fractured wall 
Similar observations can be drawn from the simulation of the same non-insulated facades during the month of july, as seen on Fig. 12. Though the consequences of cracks on the thermal performances are small, an important moisture accumulation can be seen to occur in the damaged simulation case, although the moisture content of the initial wall (continuous line) is supposed to slightly decrease during this summer month. A higher dependency of heat transfer on the damage state of materials is however to be observed in the simulation cases 2 and 2 f, i.e. walls including a hygroscopic inner insulation layer, shown on Fig. 13 and 14.

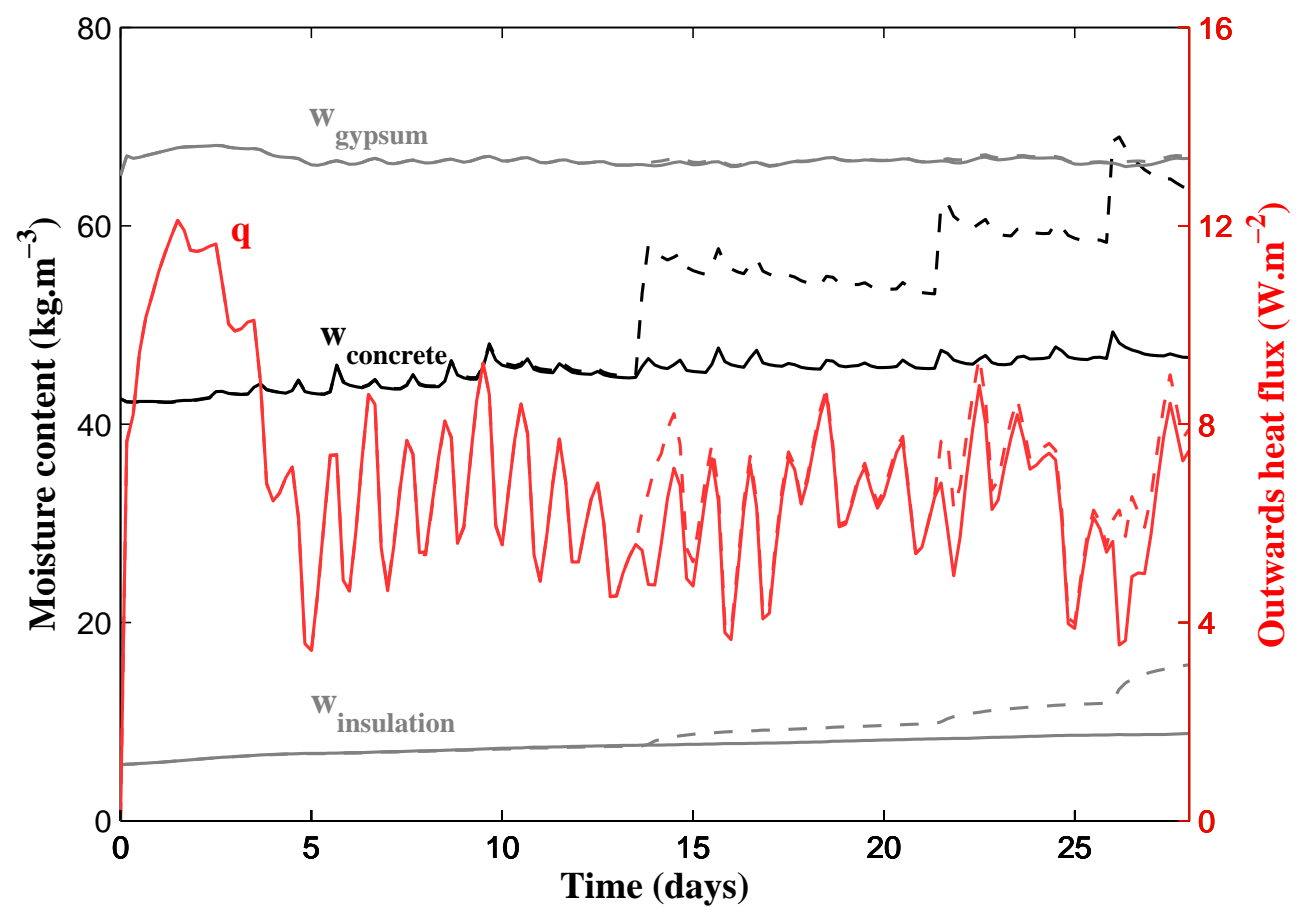

Figure 13: Wall with inner insulation (february). Continuous lines: initial wall; dotted lines: fractured wall

The behaviour of the inner-insulated facades during the winter month is displayed on Fig. 13. The presence of a fibre board between the load-bearing material and the internal finishing layer yields the following observations:

- The evolution of the moisture content of concrete, and the impact of fractures on moisture accumulation, is very similar to the case of non-insulated walls. The daily fluctuations of the gypsum board moisture content are significantly decreased, as this material is now insulated from the exterior boundary conditions.

- In the simulation case including a damaged concrete layer, the moisture content of the fibre board $w_{\text {insulation }}$ can be seen to increase after the first rain shower. Water may migrate through the cracks and reach the insulation layer. This layer then does not seem to be able to release this quantity of infiltrated water, as the value of $w_{\text {insulation }}$ does not decrease after the end of the rain events.

- Such an accumulation in the hygroscopic insulation material affects its thermal conductivity. As a consequence, the heat flow gap between intact and damaged facades is more important than in the non-insulated case, and is not only caused by water evaporation from the facade.

The same results can be drawn from the summer simulations of the inner-insulated walls. Cracks may not only aggravate the durability of damaged materials, they may also impact the thermal performance of insulated buildings. Although this impact is still low for simulation times of one month, such consequences might be important on the long term. As shown by the expression of the thermal conductivity of fibre board (see Tab. 1), the 


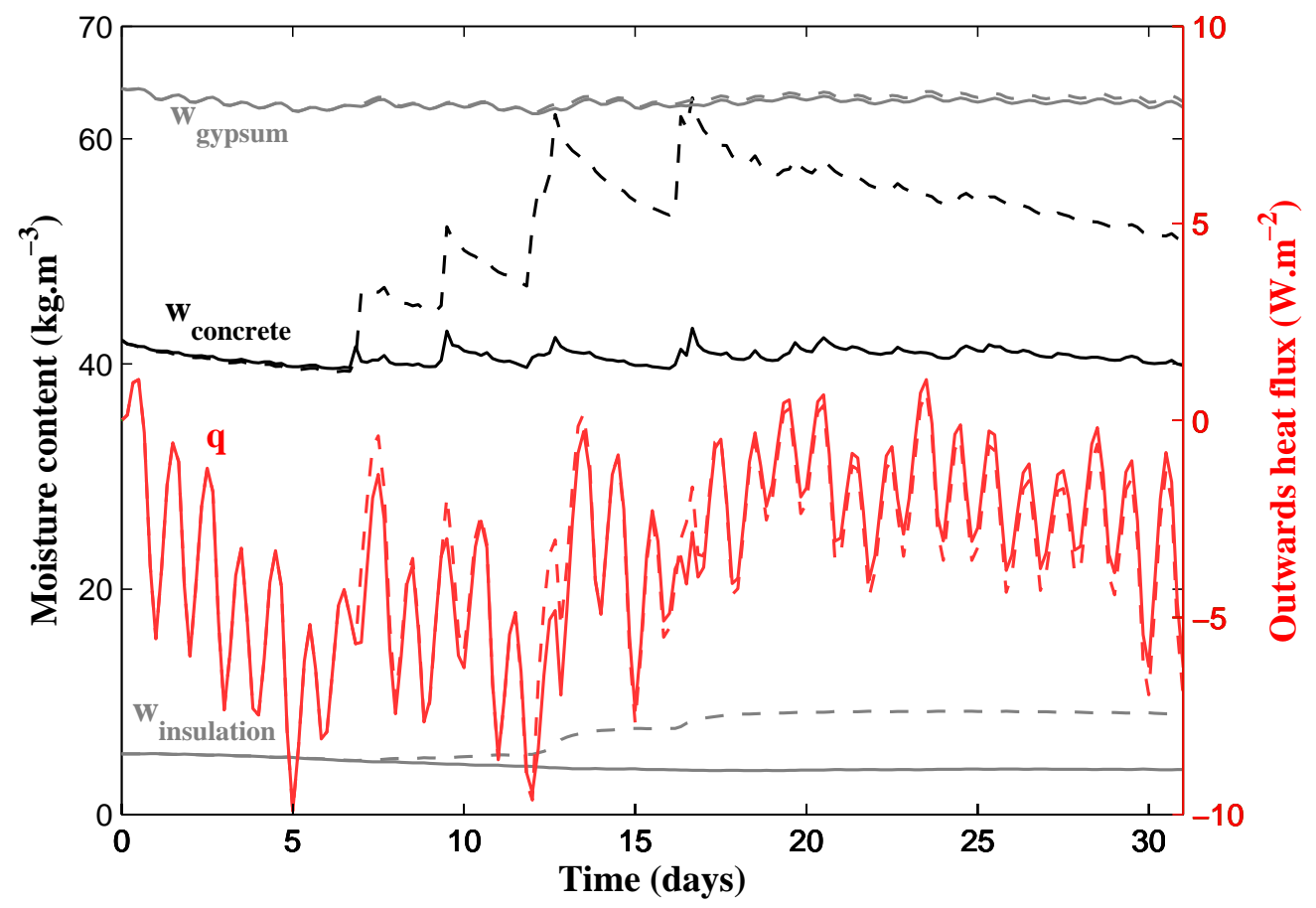

Figure 14: Wall with inner insulation (july). Continuous lines: initial wall; dotted lines: fractured wall

initial value of $\lambda$ is $0.06 \mathrm{~W} \cdot \mathrm{m}^{-1} \cdot \mathrm{K}^{-1}$, while its water-saturated value can reach $0.55 \mathrm{~W} \cdot \mathrm{m}^{-1} \cdot \mathrm{K}^{-1}$. The infiltration of rain across the load-bearing concrete, made possible by the presence of cracks, may therefore cancel the benefits of insulation after several years. Performing simulations over such longer times might be of great interest to verify this hypothesis: this has however not been performed in the present study due to a matter of calculation time.

The wall configuration $\sharp 3$, shown on Fig. 7 , is suggested as a means to correct these flaws. This configuration includes an external insulation layer, protected from climatic conditions by a hygrophobic coating. A candidate for this purpose is a previously studied engineered cementitous composite including glass fibres and a dispersed polymer phase [6]. This material has a very low permeability to liquid water and vapour. In this case, the presence of cracks in the load-bearing material no longer implies an aggravation of moisture infiltration in the wall.

It must be noted that the observations resulting from this series of simulations are given by specific cases, which have been defined in order to illustrate the potential effects of cracking on the hygrothermal performance of building components. These results are therefore to be analysed with caution. Furthermore, the procedure is based on a number of simplifying hypotheses and flaws: simplification of the climatic load, imperfect meshing strategy, restriction to 2-dimensional observation... Illustrating the potential effects of fractures however justifies the need for including mechanical considerations in hygrothermal performance assessments of building components.

\subsection{Moisture balance and durability}

The results of the monthly simulations are summarised by showing the value of the moisture balance quantities defined in Eq. 21 and 22. Fig. 15 displays the total amount of adsorbed and released moisture, per unit exposed surface of each wall, during a month of simulation.

Two main quantities can be related to a durability assessment of a building component. First, the values of $G_{\text {sorp }}$ and $G_{\text {desorp }}$ illustrate the amplitude of the average sorption/desorption cycles. Secondly, the quantity 


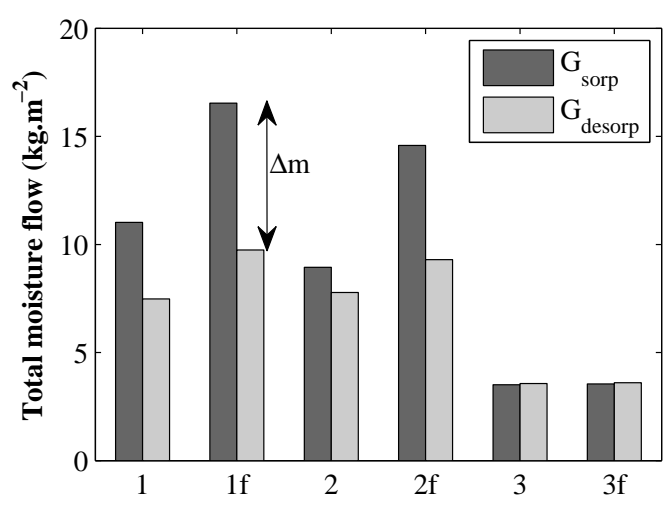

(a)

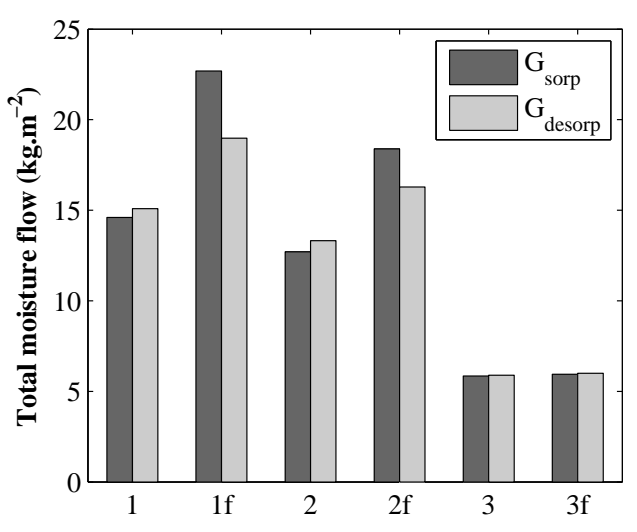

(b)

Figure 15: Total moisture sorption and desorption in all simulation cases, during (a) the winter month and (b) the summer month

$\Delta m=G_{\text {sorp }}-G_{\text {desorp }}$ (see Fig. 15(a)), illustrates moisture accumulation in the component. Both phenomena are known to accelerate degradation mechanisms: the amplitude of drying/wetting cycles accelerates the chemically induced degradation suffered by concrete [3] (alkali-silica reaction, steel reinforcement corrosion, carbonation) and accumulated water may cause frost damage in case of low temperatures, or diffuse towards the entire envelope and generate mould growth [7]. In regards to both these parameters (moisture accumulation and wetting/drying cycles), it is clear that the presence of cracks inevitably accelerates degradation processes. While this observation could be expected, the presented model allows quantifying these phenomena, allowing more advanced durability assessments. The interpretation of Fig. 15 furthermore yields the following observations:

- The aggravation of moisture accumulation due to cracking concerns both non-insulated and inner-insulated walls equally. In the second case, the infiltration of water may also reach the insulation material, should cracks form a continuous path towards this layer. This has important potential repercussions, considering such materials often do not tolerate moisture accumulation.

- During the summer month, the final moisture balance is negative in the non-damaged walls 1 and 2 ( $G_{\text {desorp }}>$ $G_{\text {sorp }}$ ), and positive in the walls including fractured components if and $2 \mathrm{f}$.

- Cracking has very little impact on the hygric performance of the outer-insulated wall (simulation cases 3 and 3f). This observation shows that the addition of a protective hygrophobic coating may correct the flaws generated by ageing, and consequently improve the durability of the structure.

Such consequences of water leakage are among the main motivations for the application of hygrothermal modelling tools. Previous examples of similar simulations integrating the complexity of climatic conditions and of multi-layered walls include projects conducted at the NRC institute [20]: the importance of indicators for the prediction of moisture-related damage was underlined.

\section{Conclusion}

The study is centered on the application of a previously developed finite-element model for heat and moisture flow simulations in fractured porous media.

- A series of simulation cases was defined in order to estimate the consequences of concrete cracking on the hygrothermal performance of non-insulated and insulated building facades. A climatic file, representative of average conditions in Lyon, was assigned to the simulations. 
- The accumulation of moisture in the wall, as well as the amplitude of the sorption/desorption cycles, can be directly visualised. This allows comparing the performance and the durability of a given wall geometry, after integrating measurements of fracture networks. Long-term consequences of cracking on the hygrothermal performance of components can therefore be estimated.

- Fractures observed in one of the material layers may influence the properties and the durability of the other layers of the facade, because of moisture infiltration. Simulations of inner-insulated walls were performed as an example of this phenomenon.

- It has been shown that the infiltration of rain, made possible by the presence of cracks in load-bearing materials, may progressively increase the moisture content of insulation layers, and thus alter their thermal properties. In some configurations, damage therefore may cancel the benefits of thermal insulation on the long term.

- The case of an outer insulation including hygrophobic coating is suggested as a means to correct the negative impact of cracking and prevent an acceleration of ageing.

Such results are however not to be expected from any material configuration or climatic conditons. Indeed, the consequences of material ageing on heat and moisture transfer are strongly dependent of the degradation process of the considered materials, and on the size and distribution of fissures and defects. The present study however showed a possible outcome of cracking on the performance of building components. Such potential effects of fractures justify the need for including mechanical considerations in hygrothermal performance assessments of building components.

The main advantage of the undertaken procedure is its applicability to a wide range of facade types. Crack geometries, integrated in the calculations, originate from experimental measurements and have no restrictions of number and sizes. Because the mechanical behaviour of the materials is not calculated, the methodology does not require the superposition of sub-systems in a staggered resolution scheme: the time-stepping procedure is simplified in comparison to fully predictive approaches. Furthermore, long-term simulations can be performed within a reasonable computational time.

\section{Acknowledgements}

The results presented in this paper have been obtained during the Renovbat project for the study of flow in fractured building components. Funding is provided by the Energy research cluster of the Rhône-Alpes region, which the authors would like to thank for their financial support through the project.

\section{References}

[1] M.O. Abadie and K.C. Mendonca. Moisture performance of building materials: From material characterization to building simulation using the moisture buffer value concept. Building and Environment, 44:388-401, 2009.

[2] J. Alfaiate, P. Moonen, L.J. Sluys, and J. Carmeliet. On the use of strong discontinuity formulations for the modeling of preferential moisture uptake in fractured porous media. Computational Methods Applied Mechanical Engineering, 199:2828-2839, 2010.

[3] C. Andrade, J. Sarría, and C. Alonso. Relative humidity in the interior of concrete exposed to natural and artificial weathering. Cement and Concrete Research, 29(8):1249-1259, 1999.

[4] B. Blocken and J. Carmeliet. A review of wind-driven rain research in building science. Journal of Wind Engineering and Industrial Aerodynamics, 92(13):1079-1130, 2004. 
[5] B. Blocken and J. Carmeliet. Guidelines for the required time resolution of meteorological input data for winddriven rain calculations on buildings. Journal of Wind Engineering and Industrial Aerodynamics, 96(5):621639, 2008.

[6] F. Chalencon, L. Orgéas, P.J.J. Dumont, G. Foray, J.-Y. Cavaillé, E. Maire, and S. Rolland du Roscoat. Lubricated compression and X-ray microtomography to analyse the rheology of a fibre-reinforced mortar. Rheologica Acta, 49:221-235, 2010.

[7] J.A Clarke, C.M Johnstone, N.J Kelly, R.C McLean, J.A anderson, N.J Rowan, and J.E Smith. A technique for the prediction of the conditions leading to mould growth in buildings. Building and Environment, 34(4):515-521, 1999.

[8] John Dolbow, Nicolas Moës, and Ted Belytschko. Discontinuous enrichment in finite elements with a partition of unity method. Finite Elements in Analysis and Design, 36(3-4):235-260, 2000.

[9] J. Grunewald. Diffusiver und konvektiver Stoff- und Energietransport in kapillar-porösen Baustoffen. PhD thesis, Technische Universität Dresden, 1997.

[10] C.-E. Hagentoft, A.S. Kalagasidis, B. Adl-Zarrabi, S. Roels, J. Carmeliet, H. Hens, J. Grunewald, M. Funk, R. Becker, D. Shamir, O. Adan, H. Brocken, K. Kumaran, and R. Djebbar. Assessment method of numerical prediction models for combined heat, air and moisture transfer in building components. benchmarks for one-dimensional cases. Journal of Thermal Envelope and Building Science, 27:327-352, 2004.

[11] C.E. Hagentoft. Hamstad wp2 - benchmark package. Technical report, Dept. of Building Physics - Chalmers University of Technology, 2002.

[12] C.E. Hagentoft. Hamstad wp2 - reference document basic modelling physics. Technical report, Dept. of Building Physics - Chalmers University of Technology, 2002.

[13] H. Janssen, B. Blocken, and J. Carmeliet. Conservative modelling of the moisture and heat transfer in building components under atmospheric excitation. International Journal of Heat and Mass Transfer, 50(5-6):11281140, 2007.

[14] M. Jiričková and R. Černý. Effect of hydrophilic admixtures on moisture and heat transport and storage parameters of mineral wool. Construction and Building Materials, 20:425-434, 2006.

[15] M.K. Kumaran, J.C. Lackey, N. Normandin, F. Tariku, and D. van Reenen. A thermal and moisture transport property database for common building and insulating materials. Technical report, ASHRAE research project 1018-RP, 2002.

[16] J. M. Melenk and I. Babuska. The partition of unity finite element method: Basic theory and applications. Computer Methods in Applied Mechanics and Engineering, 139(1-4):289 - 314, 1996.

[17] N. Moes, J. Dolbow, and T. Belytschko. A finite element method for crack growth without remeshing. International Journal for Numerical Methods in Engineering, 46:131-150, 1999.

[18] P. Moonen. Continuous-discontinuous modelling of hygrothermal damage processes in porous media. $\mathrm{PhD}$ thesis, Katholieke Universiteit Leuven, 2009.

[19] P. Moonen, L.J. Sluys, and J. Carmeliet. A continuous-discontinuous approach to simulate heat transfer in fractured media. Transport in Porous Media, 89:399-419, 2011.

[20] P. Mukhopadhyaya, K. Kumaran, F. Tariku, and D. Van Reenen. Application of hygrothermal modelling tool to assess moisture response of exterior walls. Journal of Architectural Engineering, 12:178-186, 2006.

[21] C.R. Pedersen. Combined heat and moisture transfer in building constructions. PhD thesis, Technical University of Denmark, 1990. 
[22] V. Reichenberger, H. Jakobs, P. Bastian, and R. Helmig. A mixed-dimensional finite volume method for twophase flow in fractured porous media. Advances in Water Resources, 29(7):1020-1036, 2006.

[23] J. Rethoré, R. de Borst, and M.-A. Abellan. A two-scale approach for fluid flow in fractured porous media. International Journal for Numerical Methods in Engineering, 71:780-800, 2007.

[24] S. Roels, J. Carmeliet, and H. Hens. Hamstad wp1 - moisture transfer properties and materials characterization. Technical report, K.U.Leuven - Laboratory of Building Physics, 2003.

[25] S. Roels, P. Moonen, K. De Proft, and J. Carmeliet. A coupled discrete-continuum approach to simulate moisture effects on damage processes in porous materials. Computer Methods in Applied Mechanics and Engineering, 195(52):7139-7153, 2006.

[26] S. Roels, K. Vandersteen, and J. Carmeliet. Measuring and simulating moisture uptake in a fractured porous medium. Advances in Water Resources, 26(3):237-246, 2003.

[27] S. Rouchier. Hygrothermal performance assessment of damaged building materials. $\mathrm{PhD}$ thesis, Université Claude-Bernard Lyon 1, Lyon, France, 2012.

[28] S. Rouchier, G. Foray, N. Godin, M. Woloszyn, and J.-J. Roux. Damage monitoring in fibre reinforced mortar by combined digital image correlation and acoustic emission. Construction and Building Materials, 38:371-380, 2013.

[29] S. Rouchier, G. Foray, M. Woloszyn, and J.-J. Roux. Influence of diffuse damage on the water vapour permeability of fibre-reinforced mortar. Transport in Porous Media, 93:543-559, 2012.

[30] S. Rouchier, H. Janssen, C. Rode, M. Woloszyn, G. Foray, and J.-J. Roux. Non-destructive observation of damage in mortar and concrete during mechanical loading for the evaluation of moisture transfer properties. In Proceedings of the $5^{\text {th }}$ International Building Physics Conference, Kyoto, Japan, 2012, 2012.

[31] Simon Rouchier, Hans Janssen, Carsten Rode, Monika Woloszyn, Geneviève Foray, and Jean-Jacques Roux. Characterization of fracture patterns and hygric properties for moisture flow modelling in cracked concrete. Construction and Building Materials, 34:54-62, 2012.

[32] Bernhard A. Schrefler, Stefano Secchi, and Luciano Simoni. On adaptive refinement techniques in multifield problems including cohesive fracture. Computer Methods in Applied Mechanics and Engineering, 195(46):444-461, 2006. Adaptive Modeling and Simulation.

[33] S. Secchi and L. Simoni. An improved procedure for $2 \mathrm{~d}$ unstructured delaunay mesh generation. Advances in Engineering Software, 34(4):217-234, 2003.

[34] K. Sedlbauer. Prediction of mould growth by hygrothermal calculation. Journal of Thermal Envelope and Building Science, 25:321-336, 2002.

[35] J.M. Segura and I. Carol. On zero-thickness interface elements for diffusion problems. International Journal for Numerical and Analytical Methods in Geomechanics, 28:947-962, 2004.

[36] J.M. Segura and I. Carol. Coupled $\mathrm{hm}$ analysis using zero-thickness interface elements with double nodes. Part I: theoretical model. International Journal for Numerical and Analytical Methods in Geomechanics, 32:2083-2101, 2008.

[37] J.M. Segura and I. Carol. Coupled hm analysis using zero-thickness interface elements with double nodes. Part II: verification and application. International Journal for Numerical and Analytical Methods in Geomechanics, 32:2103-2123, 2008.

[38] D. Snow. Anisotropic permeability of fractured media. Water Resources Research, 5:1273-1289, 1969. 
[39] H.-J. Steeman, M. Van Belleghem, A. Janssens, and M. De Paepe. Coupled simulation of heat and moisture transport in air and porous materials for the assessment of moisture related damage. Building and Environment, 44(10):2176 - 2184, 2009.

[40] F. Tariku, K. Kumaran, and P. Fazio. Transient model for coupled heat, air and moisture transfer through multilayered porous media. International Journal of Heat and Mass Transfer, 53:3035-3044, 2010.

[41] K. Vandersteen, J. Carmeliet, and J. Feyen. A network modeling approach to derive unsaturated hydraulic properties of a rough-walled structure. Transport in Porous Media, 50:197-221, 2003.

[42] G.N. Wells and L.J. Sluys. A new method for modelling cohesive cracks using finite elements. International Journal of Numerical Methods in Engineering, 50:2667-2682, 2001. 